\title{
Making True Amplitude Angle Domain Common Image Gathers Using Invertible Radon Transform
}

\author{
Hongwei Liu* and Yi Luo \\ EXPEC Advanced Research Center, Saudi Arabia.
}

Received 26 March 2018; Accepted (in revised version) 15 October 2018

\begin{abstract}
Converting subsurface offset domain common image gathers (ODCIGs) to angle domain common image gathers (ADCIGs) through a Radon Transform (RT) in either the spatial or wavenumber domain is efficient and valid except for the distortion of both frequency spectrum and amplitude versus angle (AVA) effect. This paper presents two modifications to the existing method to keep the frequency spectrum of the resultant ADCIGs the same as the input data and to preserve the relative amplitudes. The spectrum invariance is achieved by replacing the conventional RT or slant slack by an invertible RT. Amplitude preservation is obtained by applying an amplitude correction factor in the angle domain. Tests on both synthetic and field datasets validate the accuracy of these modifications.
\end{abstract}

AMS subject classifications: $86-08$

Key words: True amplitude, ADCIG, reverse time migration, invertible Radon transform.

\section{Introduction}

Reverse time migration (RTM) aims to construct an image of subsurface reflectors by using recorded seismic data and migration velocity models. The images can be formed by calculating numerical solutions to the two-way wave equations based on these given conditions. While the goal of conventional migration is to produce images of subsurface structure, so-called true amplitude or amplitude preserving migration attempts to maintain relative amplitude information. As a consequence, event amplitudes in the migrated results (stacked images or gathers before stacking) become proportional to the angle-dependent reflection coefficients of subsurface reflection interfaces. Zhang and Sun (2009) showed that true amplitude RTM can provide better products for subsalt imaging and AVA analysis than those from normal migration methods.

${ }^{*}$ Corresponding author. Email addresses: Hongwei.Liu@aramco.com (H. Liu), Yi.Luo@aramco.com (Y. Luo) 
An alternative method to estimate reflection coefficients is through seismic inversion, such as Least Squares Migration (LSM). Inversion techniques usually need several iterations to converge, while true amplitude RTM requires only one iteration. Indeed, true amplitude migration can be considered as the first iteration of least squares migration. Because only one iteration is employed, it is critical that this iteration is properly preconditioned for recovering the reflection coefficients; such pre-conditioning operators have been well established in the past several decades. Another advantage of migration over inversion approaches is the resistance to noise because no data matching is performed in the migration process. Given its computational efficiency and robustness to noise, true amplitude RTM has been, and will keep playing an important role for imaging subsurface structures and estimating medium properties.

Bleistein et al. (2001) derived the true-amplitude Kirchhoff migration formula, which is a by-product of his ray-based seismic inversion theory. Other researchers further developed wave-equation-based true amplitude migration methodologies, including oneway wave equation migration (Zhang et al., 2005) and RTM (Xu et al., 2011; Zhang and Sun, 2009). To gain leverage from true amplitude migration, many researchers have developed various methods to produce ADCIGs (Biondi and Symes, 2004; Dickens and Winbow, 2011; Luo et al., 2010; Sava and Fomel, 2003, 2006; Shan and Biondi, 2008; Xie and $\mathrm{Wu}, 2002$; Xu et al., 2001, 2011; Yan and Xie, 2009, 2012; Yoon et al., 2011; Zhang, et al., 2010). True amplitude ADCIGs have been used for creating better stacked images and for revealing subtle and complex geologic features.

Among the methods used for generating ADCIGs, converting the migrated images from the subsurface-offset domain to the angle domain is an easy-to-implement approach. It can be realized by performing a conventional RT in the offset-depth domain or by radial-trace mapping in the offset-depth wavenumber domain (Sava and Fomel, 2000; 2003). However, the resultant ADCIGs may have degraded accuracy in terms of preserving the true amplitude and original frequency spectrum (Jin et al., 2014; Luo et al., 2010). This paper modifies the conventional RT-based method for preserving true amplitude and frequency content. We first introduce an invertible RT, which yields better frequency spectrums than the conventional RT. Then, we derive and apply an amplitude compensation factor for better preservation of the amplitude information. The effectiveness of these techniques on improving the quality of migrated images is confirmed by synthetic and field data tests.

\section{Methods}

\subsection{Angle domain true amplitude Kirchhoff migration}

True amplitude angle-domain Kirchhoff migration (Xu et al., 2001) can be written as:

$$
\tilde{R}(x, \theta)=\int_{x_{s_{1}}}^{x_{s_{2}}} \iint i \omega \frac{\cos \left(\alpha_{s}\right) \cos \left(\alpha_{r}\right)}{v_{s} v_{r}} A_{s} A_{r} e^{i \omega\left(\tau_{s}+\tau_{r}\right)} Q d \omega d x_{r} d x_{s}
$$


In this equation, $\tilde{R}(x, \theta)$ is the angle dependent reflectivity, $\alpha_{s}$ and $\alpha_{r}$ are the propagation angles at the source and receiver locations between the vertical and ray trajectory directions, $\cos \left(\alpha_{s}\right)$ and $\cos \left(\alpha_{r}\right)$ are called obliquity factors from the source and receiver sides, $v_{s}$ and $v_{r}$ are the migration velocities at the source and receiver location, $A_{s}$ and $A_{r}$ are the Green's function amplitudes from the source and receiver locations, $\tau_{s}$ and $\tau_{r}$ are the Green's function traveltimes from the source and receiver locations to the image location, and $Q$ are the recorded seismic traces. It is important to note that the recorded traces in Eq. (2.1) are recorded data in the whole space. In reality, the data are recorded on or near the free surface of the half space and ghost waves must be taken into account. Eq. (2.1) can preserve amplitudes if the input data are recorded in a whole space, otherwise additional effort must be made to compensate the effects of free surface.

\subsection{Angle domain true amplitude RTM}

RTM calculates two wavefields including a forward propagated source wavefield and a backward propagated receiver wavefield. The temporal dot product of these two wavefields will generate desired images of subsurface reflectors. We adopt the true amplitude angle-domain RTM theory from Zhang and Sun (2009) for creating benchmarks for our proposed method. According to their method, Eqs. (2.2) and (2.3) (see below) are employed for calculating the source and receiver wavefields $S\left(x, x_{s}, \theta, t\right)$ and $R\left(x, x_{s}, \theta, t\right)$, respectively. In our implementation, both source function $f(t)$ and recorded data $Q(t)$ are injected into the wavefields computed by a finite-difference algorithm. To simulate the boundary conditions, a mirror source function $-f(t)$ and mirror recorded data $-Q(t)$ are also injected. We have chosen this way in order to avoid unwanted free-surface-related artifacts, and more importantly, the mirror source and receiver data are used to generate the obliquity factors in Eq. (2.1).

$$
\begin{aligned}
& \left\{\begin{array}{l}
\frac{1}{c^{2}} \ddot{S}=\nabla^{2} S, \\
S(x, z=0, t)=\delta(x) \int_{-\infty}^{t} f\left(t^{\prime}\right) d t^{\prime},
\end{array}\right. \\
& \left\{\begin{array}{l}
\frac{1}{c^{2}} \ddot{R}=\nabla^{2} R, \\
R(x, z=0, t)=Q(t),
\end{array}\right. \\
& R\left(x, x_{s}, \theta\right)=\int_{0}^{T_{\max }} S\left(x, x_{s}, \theta, t\right) R\left(x, x_{s}, \theta, t\right) d t, \\
& R\left(x, \theta_{0}\right)=\int R\left(x, x_{s}, \theta\right) \delta\left(\theta-\theta_{0}\right) d x_{s} .
\end{aligned}
$$

The zero-lag cross-correlation imaging condition in Eq. (2.4) is used to generate a migrated image from every shot record. The resulting image $R\left(x, x_{s}, \theta\right)$ can be further converted to the angle domain according to Eq. (2.5), assuming the incidence angles are pre-computed or provided. In the simplest case, the incidence angles can be computed 
using Pythagorean theory for a homogeneous medium. Indeed, this approach is used to generate benchmarks for testing our proposed method. One can prove that Eq. (2.5) is essentially the same as Eq. (2.1) (Zhang et al., 2005; Zhang and Sun, 2009).

\subsection{Sub-offset domain common image gather}

Sava and Fomel $(2000,2003)$ proposed a way to build extended migration images with a sub-surface spatial shift to compute a subsurface offset gather $I(h, x, z)$ :

$$
I(h, x, z)=\int_{0}^{T_{\max }} S(x-h, z, t) R(x+h, z, t) d t .
$$

The ODCIG $I(h, x, z)$ is then converted to an ADCIG by using the following RT:

$$
I_{1}(x, z, \theta)=\int_{-H}^{H} I(h, x, z-h \tan \theta) d h,
$$

where $H$ is the maximum offset. A RT is also called a slant stack in seismic data processing.

The ODCIG in Eq. (2.6) is well known and commonly used for wave equation migration velocity analysis (WEMVA), such as in differential semblance optimization (DSO) (Shen and Symes, 2008; Sava and Biondi 2004). These methods automatically update the velocity model through measuring the focusing of the ODCIGs without any picking.

\subsection{Invertible Radon transform for ADCIG}

The ADCIG from Eq. (2.7) fails to preserve the amplitude and frequency spectrum (Jin et al., 2014; Luo et al., 2010). To preserve the frequency spectrum, we apply back-projection "Rho Filtering" $|\omega|$ (Hiriyannaiah, 1997) on $I_{1}$ (Eq. (2.8)), where the convolution is based on $z$ in $I_{1}(z, \theta)$. The back-projection filter is shown in Eq. (2.9), where is the depth sampling interval. In the following section, we will use synthetic data to validate the new projection filter

$$
\begin{aligned}
& I_{2}(z, \theta)=I_{1}(z, \theta) * h(n Z), \\
& h(n Z)= \begin{cases}\frac{1}{4 Z^{2}}, & n=0, \\
0, & n \text { is even, } \\
-\frac{1}{n^{2} \pi^{2} Z^{2}}, & n \text { is odd }\end{cases}
\end{aligned}
$$

\subsection{Generation of Benchmarking datasets}

We know that reflection traveltimes from an imaginary flat interface can be modeled by direct arrivals (Fig. 1). Since there is no actual interface, the reflection coefficients 


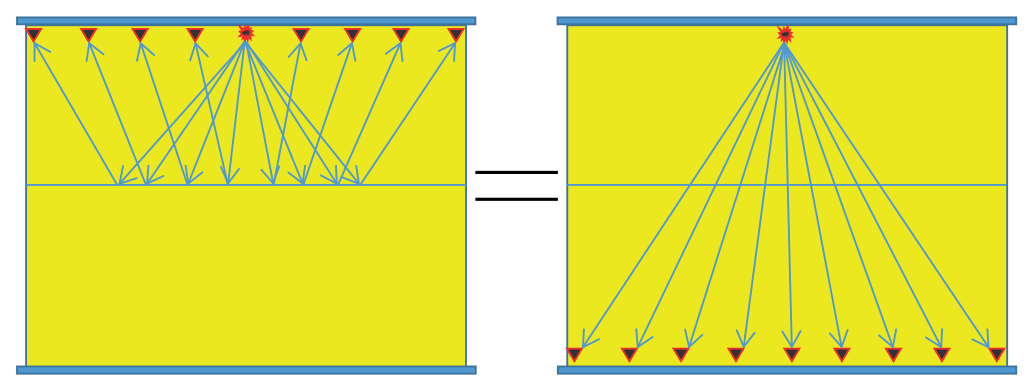

Figure 1: The method of generating benchmarking synthetic data containing reflections from a virtual interface. In this method, all four boundaries should be absorbing.

from the virtual interface are constant for any incidence angle. This virtual reflection is particularly useful for testing true amplitude RTM where the amplitudes in a migrated ADCIG are independent of the incidence angle. In the virtual reflector tests, we use absorbing boundary conditions to simulate wave propagation in an infinite space.

We simulate five virtual interfaces to perform ADCIG tests. Fig. 2(a) is common shot gather computed by a finite difference solution to the acoustic wave equation. The background velocity is $3000 \mathrm{~m} / \mathrm{s}$ and the grid size is $10 \mathrm{~m}$ in both horizontal and vertical directions. A Ricker wavelet with a $10 \mathrm{~Hz}$ peak frequency is used as the source function and five virtual interfaces from $2 \mathrm{~km}$ to $6 \mathrm{~km}$ deep are simulated using the method. We will use this dataset to test the angle domain true amplitude RTM. Fig. 2 shows that using the true velocity, the energy focuses at the correct depths at the zero offset location in the ODCIG. Fig. 3(a) is the benchmark ADCIG generated using Eq. (2.5) and Pythagorean theory using Fig. 3(c) as input. We can compare an ADCIG converted from ODCIG using a conventional forward RT (Eq. (2.7)) and an ADCIG converted using an invertible RT according to Eqs. (2.8) and (2.9). The stacks of the ADCIGs are shown in Fig. 4(a), 4(c) and 4(e), respectively. The corresponding spectra, displayed in Fig. 4(b), 4(d) and 4(f), are quite different. The frequency spectrum of the benchmark result is nearly identical as the one made from the invertible RT. It tells us that the ADCIG generated by the invertible RT can better preserve the frequency spectrum.

\subsection{Compensation of amplitude for ADCIG}

Although the frequency spectrum of the ADCIG made from invertible RT is preserved, the true amplitude capability of the ADCIG remains to be verified. Arntsen et al. (2010) pointed out that Eqs. (2.6) and (2.7) cannot create true amplitude ADCIGs and he solved this problem by changing the source of the downgoing wavefield resulting in a trueamplitude cross-correlation type imaging condition. In this paper, to preserve the amplitude of the ADCIG, we will apply angle binning during the invertible RT. As shown in Fig. 2(b), the green line is the slant stack path for incidence angle $\theta$, and the two yellow triangles show the areas included in the given angle bin ( $\delta \theta$ is the binning range). 


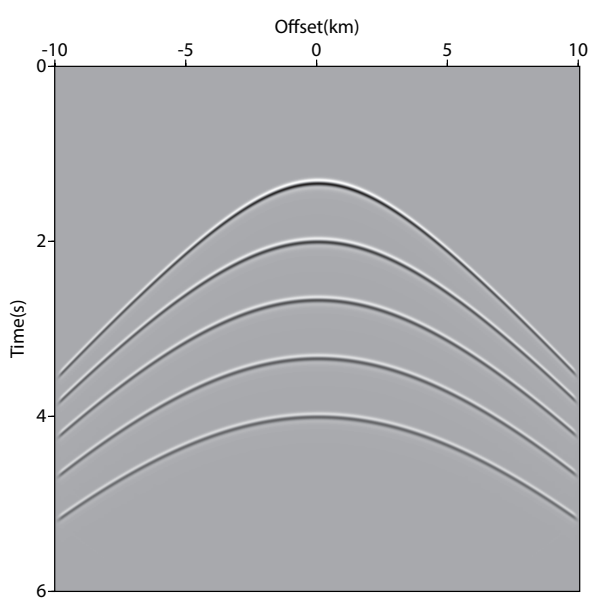

(a)

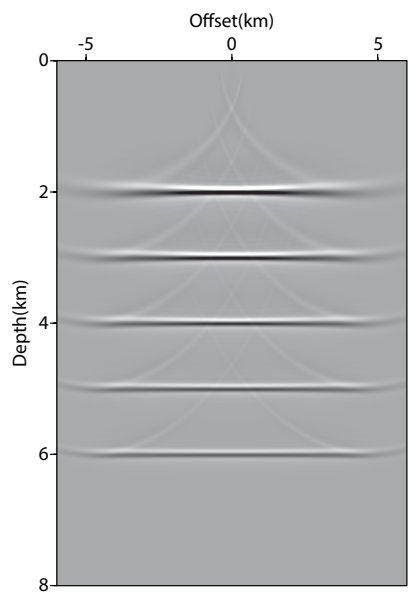

(b)

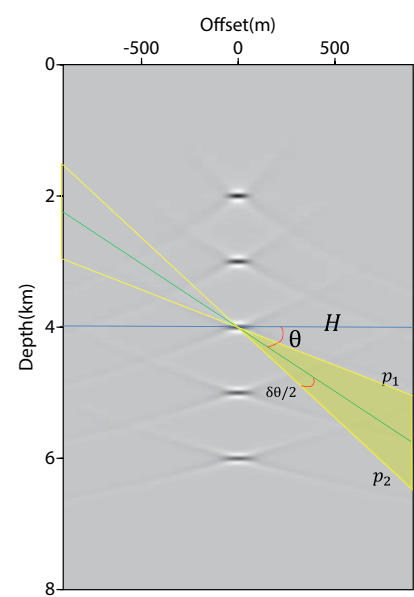

(c)

Figure 2: The benchmarking synthetic datasets containing 5 reflections generated from virtual interface methods including (a) the recorded seismic traces, (b) the migration result of one shot using Eqs. (2.2) to (2.4), and (c) the ODCIG computed from Eq. (2.6).

To apply angle binning during RT, Eq. (2.7) is replaced by the following:

$$
I_{1}^{\prime}(z, \theta)=\int_{-H}^{H} \int_{p_{2}}^{p_{1}} I(h, z+p h) d p d h,
$$

where $p_{1}=-\tan (\theta-\delta \theta / 2), p_{2}=-\tan (\theta+\delta \theta / 2)$ and $p_{1}=-\tan (\theta)$. Substituting $p, p_{1}$ and $p_{2}$ to Eq. (2.10), we can derive the following (see the Appendix):

$$
I_{1}^{\prime}(z, \theta)=\frac{\delta \theta}{(\cos \theta)^{2}} I_{1}(z \theta) .
$$

We name $\frac{\delta \theta}{(\cos \theta)^{2}}$ the amplitude compensation factor. Now we apply the amplitude compensation factor to the ADCIG $I_{2}$ generated by the invertible RT to the virtual interface 


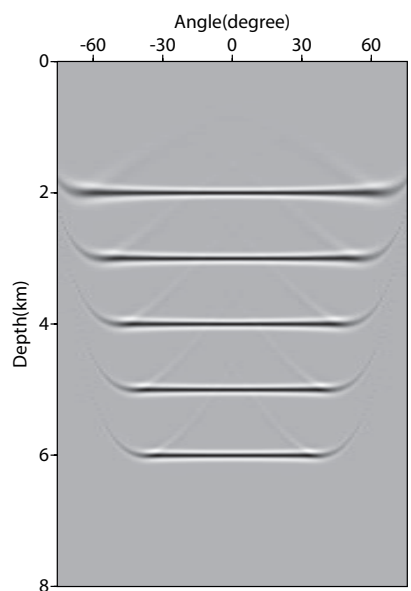

(a)

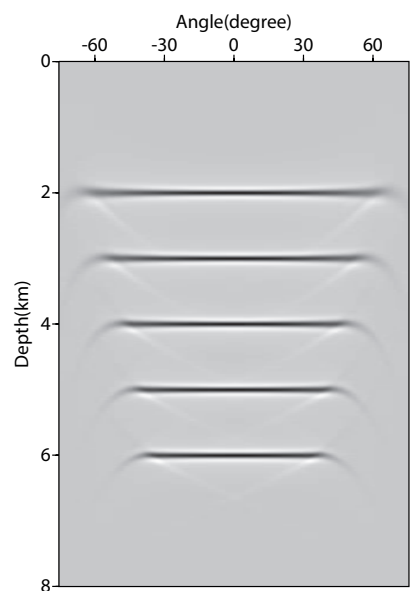

(b)

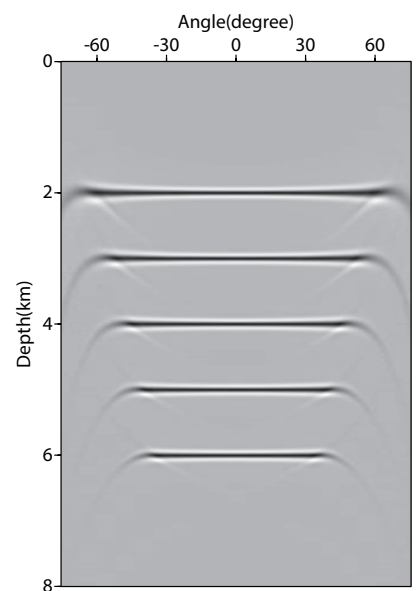

(c)

Figure 3: Synthetic tests showing ADCIGs from (a) the binning result from Fig. 2(c) using Eq. (2.5), (b) a forward RT, and (c) after applying an invertible RT and amplitude compensation factor.

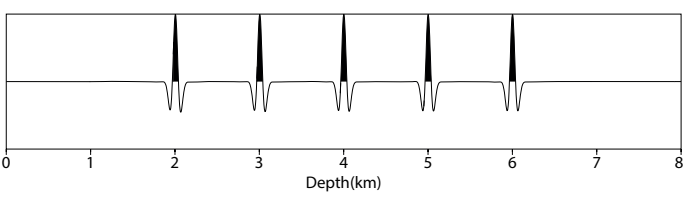

(a)

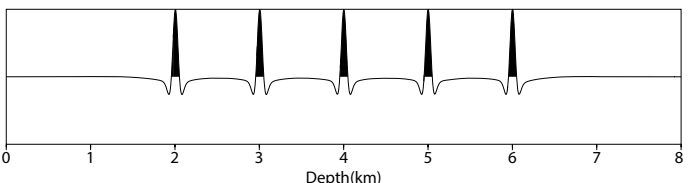

(c)

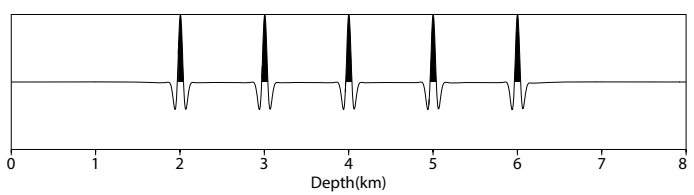

(e)

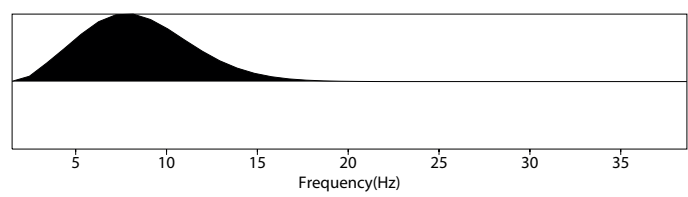

(b)

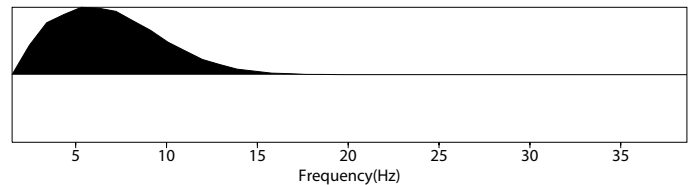

(d)

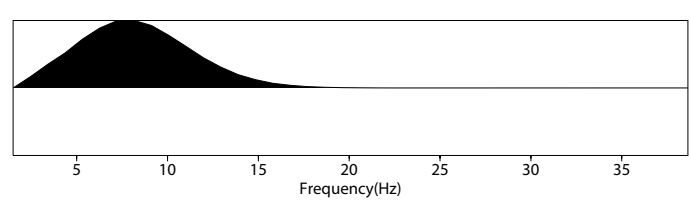

(f)

Figure 4: ADCIG stacks and frequency spectra. Panels (a), (c) and (e) are stacks of Fig. 3(a), 3(b) and 3(c), respectively, while (b), (d) and (f) are the corresponding frequency spectra.

synthetic tests. Fig. 5(c) shows the peak amplitude of the five virtual interfaces picked from Fig. 3(c). The amplitude is decaying with increasing incidence angle. After applying the amplitude compensation factor, the amplitude variation becomes flat with respect to varying incidence angle. This result is also consistent with the benchmark shown in Fig. 5(b). 


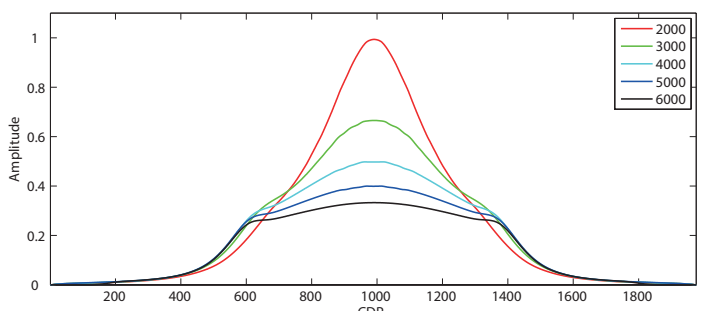

(a)

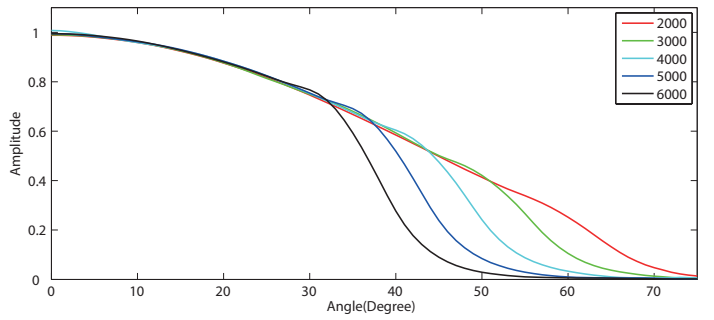

(c)

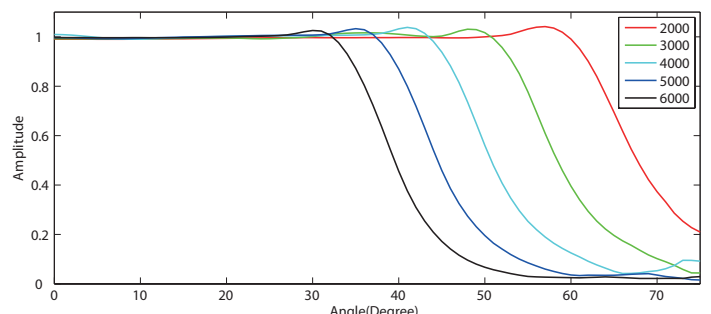

(b)

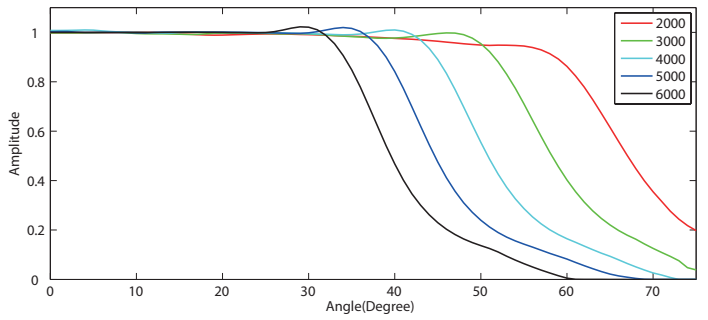

(d)

Figure 5: Peak amplitude picked from different domain migration results including those (a) picked from shot domain RTM result of Fig. 2(c), (b) from angle domain RTM of Fig. 3(a), and (c) and (d) from the angle gather before (Fig. 3(b)) and after (Fig. 3(c)) amplitude compensation, respectively.

\section{Numerical examples}

The first example is a two-layer model containing a flat interface. The upper layer velocity $V_{1}$ is $3.464 \mathrm{~m} / \mathrm{s}$ and the lower layer velocity $V_{2}$ is $4.000 \mathrm{~m} / \mathrm{s}$. A 2D finite-difference forward modeling algorithm is used to generate the synthetic data. In this example, the analytical reflection coefficient is given by Eq. (3.1) (where $\theta_{1}$ is incidence angle and $\theta_{2}$ is refracted angle) and shown by the red line in Fig. 7. Fig. 6 shows AVO variations on ADCIGs after shot to angle conversion computed using Eqs. (2.2) to (2.5) and after using the invertible RT with and without amplitude compensation. Actual amplitudes plotted versus angle are shown in Fig. 7. The result from our method matches perfectly with the analytical solution up to 50 degrees, while the conventional RT method is only accurate up to 10 degrees

$$
R\left(\theta_{1}\right)=\frac{V_{2} \cos \theta_{1}-V_{1} \cos \theta_{2}}{V_{2} \cos \theta_{1}+V_{1} \cos \theta_{2}}
$$

\subsection{BP 2D synthetic model test}

The second example uses the 2004 BP 2D synthetic dataset. Fig. 8(a) shows the stacked image using the conventional RT method whereas Fig. 8(b) is the result after the frequency and amplitude compensation. The comparison shows that after the compensation, the frequency spectrum is increased and the image quality is largely improved because of lower frequency noise is suppressed. Fig. 8(c) and 8(d) are ADCIGs extracted 


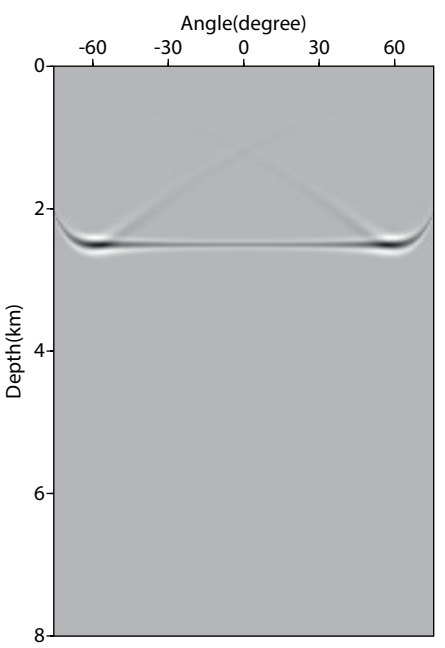

(a)

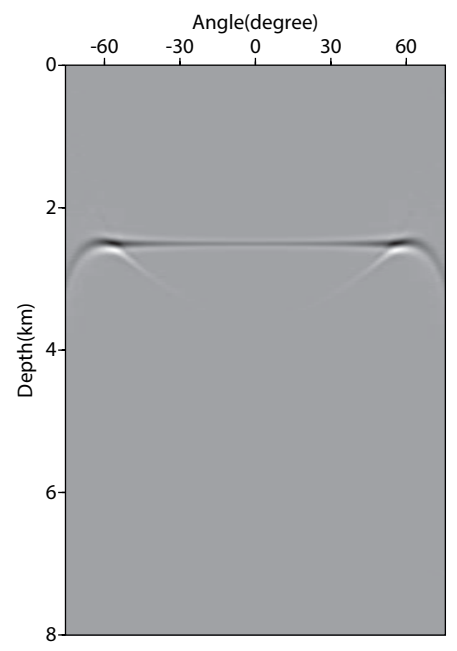

(b)

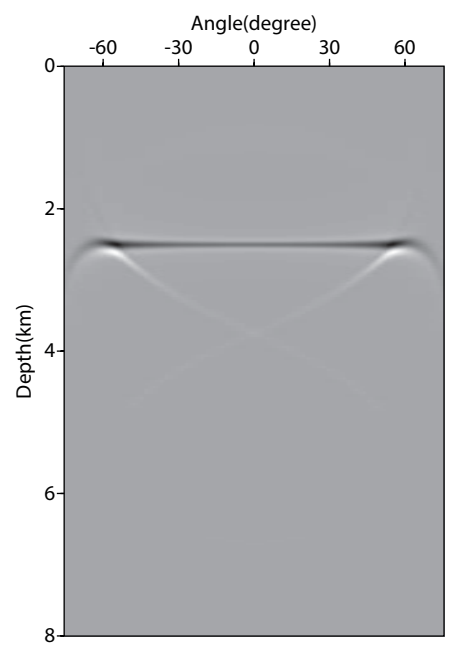

(c)

Figure 6: ADCIG tests showing (a) binning result from using Eqs. (2.2) to (2.5), (b) ADCIG using invertible RT and amplitude compensation, and (c) ADCIG using forward RT without amplitude compensation.

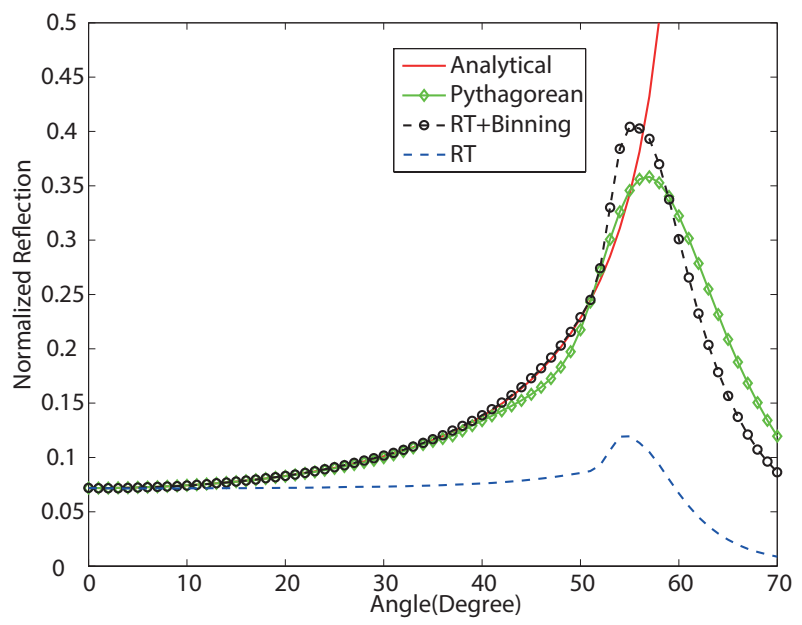

Figure 7: Reflection coefficients versus incident angle.

from Fig. 8(a) and $8(b)$ at $c d p=3000$, respectively. Again, the gather using an invertible RT is much cleaner because the frequency issue is correctly addressed and more accurate amplitude compensation is applied.

\subsection{Real data test}

The third example is an application to marine seismic data. Fig. 9(a) shows the stacked image using the conventional RT method compared to the result after frequency and 


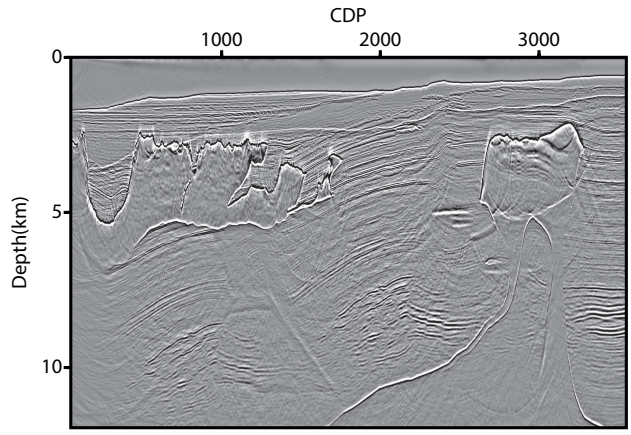

(a)

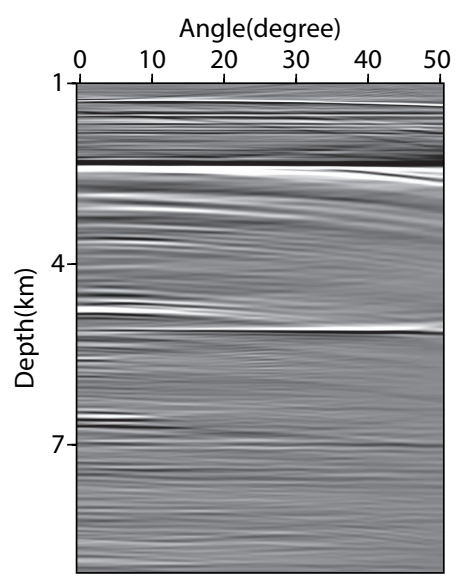

(c)

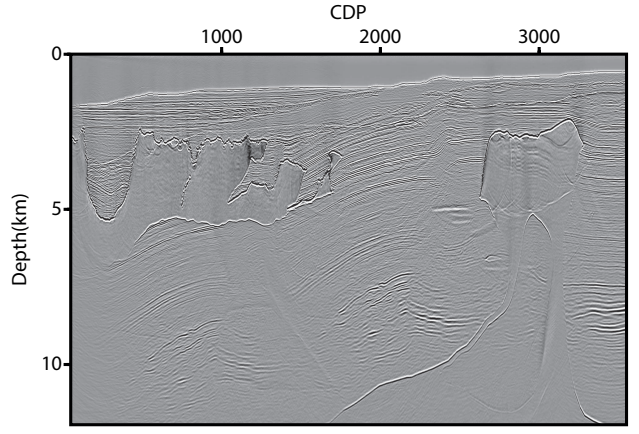

(b)

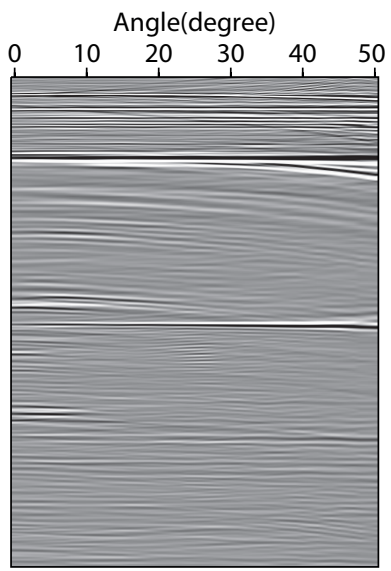

(d)

Figure 8: Tests on the BP 2004 synthetic model including the stacked image of (a) conventional and (b) invertible $\mathrm{RT}$, and $\mathrm{ADCIG}$ from $\mathrm{CDP}=3000$ using (c) a conventional RT and (d) an invertible RT.

amplitude compensation shown in Fig. 9(b). If we look at a zoom in (Fig. 9(c) and 9(d)) we can see that the resolution using the new algorithm is enhanced and low frequency noise is suppressed. The same results are seen when comparing the ADCIGs (Fig. 9(e) and $9(\mathrm{f}))$. In this test, 0 to 60 degree incidence angles are used to make the stacked image from the ADCIGs and no other denoising technique is applied.

\section{Conclusions}

We described two modifications to the widely used RT method for converting ODCIGs to ADCIGs for preserving migration amplitudes and frequency spectra using RTM. The frequency spectra are preserved when a conventional RT (or slant slack) is replaced by an invertible RT (Rho-filtering). Moreover, an amplitude compensation factor is introduced to make the amplitudes in ADCIGs proportional to the reflection coefficients. These enhancements are verified on both synthetic and real data examples. By correctly address- 


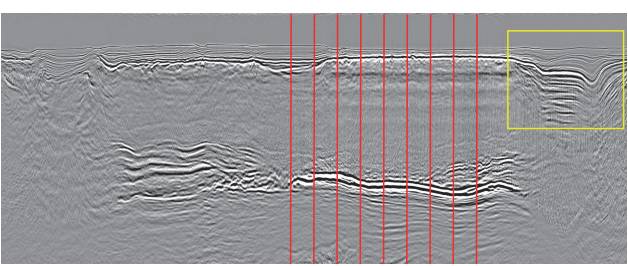

(a)

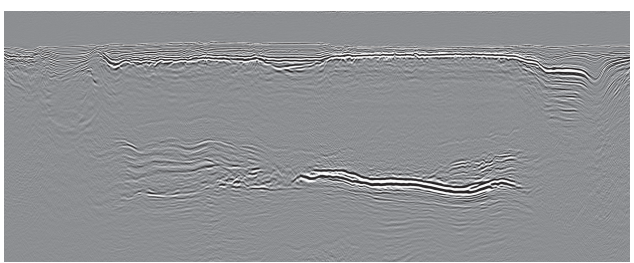

(b)

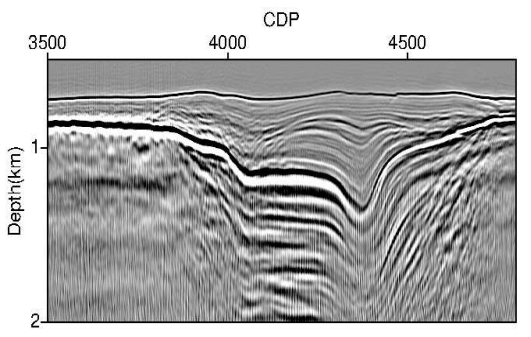

(c)

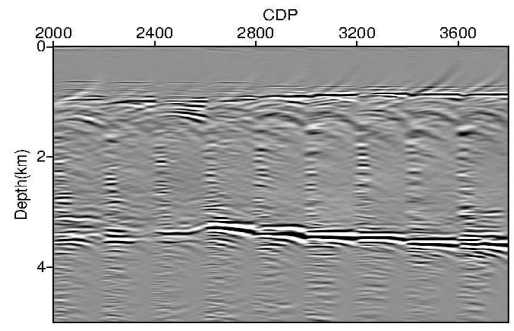

(e)

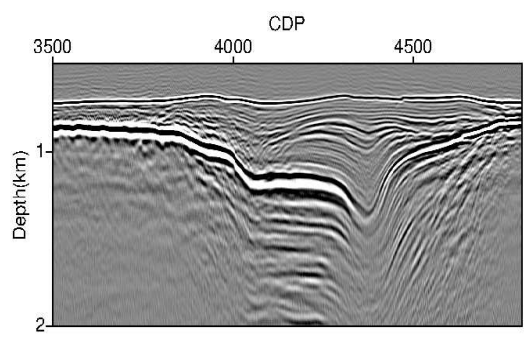

(d)

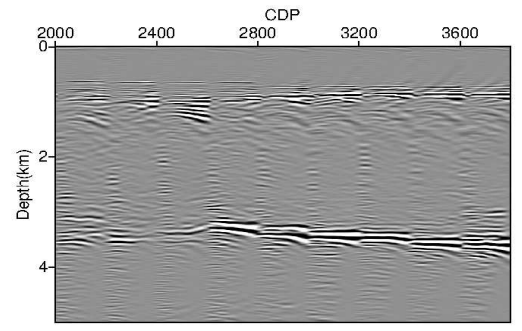

(f)

Figure 9: Real data example showing (a) a stacked image using the conventional RT method; (b) a stacked image after frequency and amplitude compensation; (c) and (d) zoom in of (a) and (b), respectively, (yellow rectangle in (a)); (e) and (f) ADCIGs using conventional and invertible RT, respectively (located at red lines in (a)).

ing amplitude and frequency spectrum information, the image quality is significantly improved.

\section{Acknowledgments}

We thank Ali Momin, Andrey Bakulin and Michael Jervis for valuable suggestions on writing this article. We also thank Saudi Aramco for permission to publish this work. 


\section{Appendix: Derivation of amplitude compensation factor}

As shown in Fig. 2(c), the green line is the slant stack path for incidence angle $\theta$, and the two yellow triangles show the areas included into the given angle bin ( $\delta \theta$ is the binning range), the ray parameters $p, p_{1}$ and $p_{2}$ are expressed as follows:

$$
\begin{aligned}
& p=-\tan \theta, \\
& p_{1}=-\tan (\theta-\delta \theta / 2), \\
& p_{2}=-\tan (\theta+\delta \theta / 2) .
\end{aligned}
$$

By substituting these ray parameters into Eq. (2.10), one can derive:

$$
\begin{aligned}
I(z, \theta) & =\int_{-h}^{h} \int_{p_{2}}^{p_{1}} I(h, z+p h) d p d h \\
& =\int_{-h}^{h} \int_{\theta+\delta \theta / 2}^{\theta-\delta \theta / 2} I\left(h, z-h \tan \theta^{\prime}\right) d\left(-\tan \theta^{\prime}\right) d h \\
& =\int_{-h}^{h} \int_{\theta-\delta \theta / 2}^{\theta+\delta \theta / 2} \frac{1}{\left(\cos \theta^{\prime}\right)^{2}} I\left(h, z-h \tan \theta^{\prime}\right) d \theta^{\prime} d h \\
& =\int_{\theta-\delta \theta / 2}^{\theta+\delta \theta / 2} \frac{1}{\left(\cos \theta^{\prime}\right)^{2}} I_{1}\left(z, \theta^{\prime}\right) d \theta^{\prime}=\frac{\delta \theta}{(\cos \theta)^{2}} I_{1}(z, \theta),
\end{aligned}
$$

$\frac{\delta \theta}{(\cos \theta)^{2}}$ is the amplitude compensation factor used in Eq. (3.1).

\section{References}

[1] Arntsen, B., Tantserev, E. and Amundsen, L., True-amplitude Cross-correlation Shot-profile Imaging Condition, 80th Annual International Meeting, SEG, Expanded Abstracts, 2010, pp. 3273-3277, doi: 10.1190/1.3513527.

[2] Biondi, B. and Symes, W. W., Angle-domain common-image gathers for migration velocity analysis by wavefield-continuation imaging, Geophysics, Vol. 69, 2004, pp. 1283-1298, doi: 10.1190/1.1801945.

[3] Bleistein, N., Cohen, J. K. and Stockwell, J. W., Mathematics of multidimensional seismic inversion: Springer, 2001.

[4] Dickens, A. and Winbow, G. A., RTM angle gathers using Poynting vectors, 81st Annual International Meeting, SEG, Expanded Abstracts, 2011, pp. 3109-3113, doi: 10.1190/1.3627841.

[5] Hiriyannaiah, H.P., X-ray computed tomography for medical imaging. IEEE signal processing magazine, Vol 14, 1997, pp. 42-59.

[6] Jin, H., McMechan, G. A. and Guan, H., Comparison of methods for extracting ADCIGs from RTM, Geophysics, Vol, 79, 2014, pp. S89-S103, doi: 10.1190/GEO2013-0336.1.

[7] Luo, M., Lu R., Winbow, G. and Bear, L., A comparison of methods for obtaining local image gathers in depth migration, 80th Annual International Meeting, SEG, Expanded Abstracts, 2010, pp. 3247-3251, doi: 10.1190/1.3513522.

[8] Sava, P. and Fomel, S., Angle-gathers by Fourier transform, Stanford Exploration Project Report, Vol 103, 2000, pp. 119-130. 
[9] Sava, P. and Fomel, S., Angle-domain common-image gathers by wavefield continuation methods: Geophysics, Vol 68, 2003, pp. 1065i-1074, doi: 10.1190/1.1581078.

[10] Sava, P. and Biondi, B., Wave-equation migration velocity analysis-I: Theory: Geophysical Prospecting, Vol 52, 2004, pp. 593-607, doi: 10.1111/j.1365-2478.2004.00447.x.

[11] Sava, P. and Fomel, S., Time-shift imaging condition in seismic migration: Geophysics, Vol 71, 2006, pp. S209-S217, doi: 10.1190/1.2338824.

[12] Shan, G. and Biondi, B., Angle-domain common-image gathers for steep reflectors, 78th Annual International Meeting, SEG, Expanded Abstracts, 2008, pp. 3068-3072, doi: $10.1190 / 1.3063982$.

[13] Shen, P. and Symes, W. W., Automatic velocity analysis via shot profile migration, Geophysics, Vol 73, 2008, pp. VE49-VE59, doi: 10.1190/1.2972021.

[14] Xie, X. and $\mathrm{Wu}, \mathrm{R}$. S., Extracting angle domain information from migrated wavefields, 72nd Annual International Meeting, SEG, Expanded Abstracts, 2002, pp. 1360-1363, doi: $10.1190 / 1.1816910$.

[15] Yan, R. and Xie, X., A new angle-domain imaging condition for reverse time migration, 79th Annual International Meeting, SEG, Expanded Abstracts, 2009, pp. 2784-2788, doi: $10.1190 / 1.3255427$.

[16] Yan, R. and Xie, X., An angle-domain imaging condition for elastic reverse time migration and its application to angle gather extraction, Geophysics, Vol 77, 2012, pp. S105-S115, doi: 10.1190/geo2011-0455.1.

[17] Xu, S., Chauris, H., Lambar, G. and Noble, M., Common angle migration: A strategy for imaging complex media, Geophysics, Vol 66, 2001, pp. 1877-1894, doi: 10.1190/1.1487131.

[18] Xu, S., Zhang, Y. and Tang, B., 3D angle gathers from reverse time migration, Geophysics, Vol 76, 2011, pp. S77-S92, doi: 10.1190/1.3536527.

[19] Yoon, K., Guo, M., Cai, J. and Wang, B., 3D RTM angle gathers from source wave propagation direction and dip of reflector, 81st Annual International Meeting, SEG, Expanded Abstracts, 2011, pp. 1057-1060, doi: 10.1190/1.3627847.

[20] Zhang, Y., and Sun, J., Practical issues of reverse time migration: true-amplitude gathers, noise removal and harmonic-source encoding, First Break, Vol 26, 2009, pp. 19-25, doi: 10.3997/1365-2397.2009002.

[21] Zhang, Y., Zhang, G. and Bleistein, N., Theory of true-amplitude one-way wave equations and true-amplitude common-shot migration, Geophysics, Vol 70, 2005, pp. E1-E10, doi: 10.1190/1.1988182.

[22] Zhang, Y., Xu, S., Tang, B., Bai, B., Huang, Y. and Huang, T., Angle gathers from reverse time migration, The Leading Edge, Vol 29, 2010, pp. 1364-1371, doi: 10.1190/1.3517308. 